\title{
A eritropoietina humana recombinante: uma breve revisão com ênfase no processo de controle da qualidade
}

\author{
The recombinant human erythropoietin: \\ a brief review with emphasis in the process of \\ quality control
}

Michele Cardoso do Nascimento ${ }^{1}$ Clarice Lima do Canto Abreu Rodrigo Netto Costa ${ }^{3}$ Wlamir Corrêa de Moura ${ }^{4}$ Isabella Fernandes Delgado 5
Recebido em: 20/07/2012

Aprovado em: 30/04/2013

1 Mestranda do Programa de Pós-graduação em Vigilância Sanitária, Analista da Qualidade, Instituto Nacional de Controle de Qualidade em Saúde/FIOCRUZ.

2 Mestre em Ciências, Tecnologista Pleno em Saúde Pública, Departamento de Imunologia, Instituto Nacional de Controle de Qualidade em Saúde/FIOCRUZ.

3 Mestre em Saúde Pública, Tecnologista Pleno em Saúde Pública, Departamento de Imunologia, Instituto Nacional de Controle de Qualidade em Saúde/FIOCRUZ.

4 Doutor em Ciências, Tecnologista Sênior em Saúde Pública, Departamento de Imunologia, Instituto Nacional de Controle de Qualidade em Saúde/FIOCRUZ.

5 Doutora em Ciências, bolsista de Produtividade do CNPq e Vice-Diretoria de Pesquisa, Ensino e Projetos Estratégicos do Instituto Nacional de Controle de Qualidade em Saúde/FIOCRUZ.

\section{Resumo}

A eritropoietina (EPO) é um hormônio glicoproteico que regula a eritropoiese, atuando sobre as células progenitoras eritroides na medula óssea. É produzido nos adultos principalmente no córtex renal. O Ministério da Saúde, em 2005, passou a fornecer produtos biológicos, dentre eles a rhEPO. A produção desses medicamentos proporcionou uma economia de $40 \mathrm{mi}-$ lhões por ano ao país. A rhEPO é produzida em células de ovário de hamster chinês - CHO. É uma glicoproteína composta de 165 aminoácidos. A massa molecular da estrutura glicosilada, que contém cerca de $40 \%$ de carboidratos, é de 30-34 kDa. A rhEPO é um medicamento que faz parte do Componente Especializado de Assistência Farmacêutica do Ministério da Saúde, como parte da Política Nacional de Assistência Farmacêutica. O INCQS recebeu, de 2007 a 2012, um total de 779 lotes de rhEPO para análise, 88\% desse total corresponde à apresentação de $4000 \mathrm{UI} / \mathrm{mL}$.

Palavras-chave: Eritropoietina. Vigilância sanitária. Controle da qualidade. Biofármacos.

\section{Abstract}

The erythropoietin (EPO) is a glycoprotein hormone wich controls the erythropoiesis, acting on erythroid progenitors cells on bone narrow. It is produced, primarily in the kidney cortex. The Ministry of Health, since 2005 provides biopharmaceuticals, including rhEPO. The production of these medicines provided an economy of 40 million per year to the country. The rhEPO is produced in Chinese Hamster Ovary cells - CHO. Is a glycoprotein composed of 165 amino acid, the molecular mass of glycosylated structure, that contains about $40 \%$ of carbohydrate, is $30-34 \mathrm{kDa}$. The rhEPO is a medicine that makes part of the Specialized Pharmaceutical Services Component, of Ministry of Health as part of the National Policy of Pharmaceutical Assistance. The INCQS received, from 2007 to 2012, a total of 779 batches of rhEPO for analysis, $88 \%$ of which corresponds to the presentation of $4000 \mathrm{IU} / \mathrm{ml}$.

Keywords: Erythropoietin. Health surveillance. Quality control. Biopharmaceuticals 


\section{Introdução}

A eritropoietina (EPO) é um hormônio glicoproteico que tem como função primordial a regulação da eritropoiese, atuando primariamente sobre as células progenitoras eritroides na medula óssea (SCHMIDT et al., 2003). A clonagem do gene da EPO humana, em 1983, permitiu a sua produção em larga escala e, posteriormente, o uso clínico (ESCHBACH et al., 1987; ELLIOTT; PHAM; MACDOUGALL, 2008). Assim, a eritropoietina humana recombinante (rhEPO) vem sendo amplamente utilizada na prática clínica para reduzir a necessidade de transfusão sanguínea em processos cirúrgicos, aumentar os níveis de hemoglobina (FARIS; RITTER; ABELS, 1996) e no tratamento de anemias de várias etiologias, incluindo anemia devido à doença renal crônica, anemia relacionada ao câncer ou ao tratamento dele. (RIZZO et al., 2001; ELLIOTT; PHAM; MACDOUGALL, 2008).

Diante dessa demanda, a garantia da segurança, não só na prática clínica, mas também no processo de produção e de controle da qualidade da rhEPO é indispensável. Um conjunto de métodos físico-químicos, microbiológicos e biológicos, entre eles a avaliação da potência biológica da rhEPO, deve ser realizado para garantir a qualidade dos produtos que são ofertados à população (GILD et al., 1996). Segundo diretrizes nacionais e internacionais, esses ensaios devem ser realizados pelo próprio fabricante a cada lote produzido para posterior liberação de uso do produto à população. No Brasil, desde 2006, além de ser avaliado pelo próprio produtor, cada lote de rhEPO utilizado pelo Ministério da Saúde é também avaliado pelo Instituto Nacional de Controle de Qualidade em Saúde (INCQS), e, somente após emissão de laudo de análise desse Instituto, os lotes produzidos são liberados para consumo humano.

Nesse contexto, o presente trabalho pretende contribuir para o conhecimento na área de avaliação da qualidade de produtos biológicos a partir de uma revisão sistemática da literatura científica sobre a rhEPO, assim como a análise de alguns resultados do INCQS referentes ao controle da qualidade dos lotes desse produto adquiridos pelo Ministério da Saúde brasileiro nos últimos seis anos.

\section{Metodologia}

Como processo metodológico, foi realizada uma revisão sistemática da literatura científica na qual pesquisaram-se as bases de dados PubMed, LILACS e SciELO, utilizando-se as palavras-chaves: eritropoietina, vigilância sanitária, controle da qualidade e biofármacos, tanto em português quanto em inglês.

\section{A eritropoietina humana}

Carnot e Deflandre, em 1906, postularam a existência de um fator humoral de regulação da eritropoiese, o qual denominaram hemopoietina, e que, segundo esses autores era produzido em resposta à anemia. Essa afirmação não foi inicialmente aceita, apesar de estar correta, devido à ausência de comprovação experimental (ZANICHELLI et al., 1995; METCALF, 2008). As pesquisas continuaram e, entre as décadas de 1940 e 1950, muitos experimentos com animais foram realizados gerando dados que reforçaram a ideia e demonstraram que esse fator humoral de regulação da formação de células vermelhas não só existia como também estava presente em níveis altos no plasma anêmico e na urina. Esse regulador foi então chamado de eritropoietina (PLZAK et al., 1955).

A EPO é um hormônio glicoproteico essencial à vida, que tem como função primordial a regulação da eritropoiese (SCHMIDT et al., 2003). Camundongos com deleções no gene da EPO ou do seu receptor (EPO-R) morrem devido à anemia ainda na vida intrauterina (WEISS, 2003).

Esse hormônio é produzido no indivíduo adulto principalmente pelas células peritubulares fibroblásticas do interstício do córtex renal e também, em menor quantidade, por células hepáticas. No feto e em recém-natos, é produzido no fígado (JACOBSON et al., 1957; FRIED, 1972; ZANJANI et al., 1981; KOURY et al., 1993; FISHER et al., 1996). A ausência ou diminuição desse hormônio na corrente sanguínea pode gerar anemia severa (KAUSHANSKY; KIPPS, 2005).

A EPO possui meia-vida de aproximadamente cinco horas, o que requer uma taxa de produção em torno de $2 \mathrm{UI} / \mathrm{Kg} /$ dia. A produção da EPO é constante; alguns 
fatores, como níveis de ferro, status nutricional, condições ambientais, estados patológicos e fatores genéticos podem afetar os níveis de EPO circulante (ELLIOTT; PHAM; MACDOUGALL, 2008).

A EPO é regulada por um complexo sistema de retroalimentação, no qual sensores renais detectam alterações no nível de oxigênio sanguíneo (KAUSHASKY; KIPPS, 2005; FRIED, 2009). Nos rins, a hipóxia gera um aumento da expressão de EPO, estimulada pelo fator induzido por hipóxia (HIF-1, do inglês hypoxia induced factor), o qual medeia a secreção da EPO bem como a regulação do seu receptor (WEISS, 2003; WANG; SEMENZA, 1993).

A EPO é secretada no plasma e, ao chegar à medula óssea, liga-se a receptores específicos na superfície de células progenitoras eritroides, estimulando assim, a diferenciação delas em hemácias (Figura 1). Ela atua como fator hormonal de estimulação mitótica e diferenciação, aumentando a formação de eritrócitos maduros a partir das células progenitoras eritroides (WEISS, 2003; FRIED, 2009).

Em 1990, pesquisadores demonstraram que o EPO-R é expresso em vários tecidos não hematopoiéticos, como endotélio, células neurais e placenta (RIBATTI, 2008). Alguns estudos têm demonstrado ainda que a EPO apresenta atividade antiapoptótica (MINAMINO; KITAKAZE, 2006).

Figura 1 - Mecanismo de ação da EPO

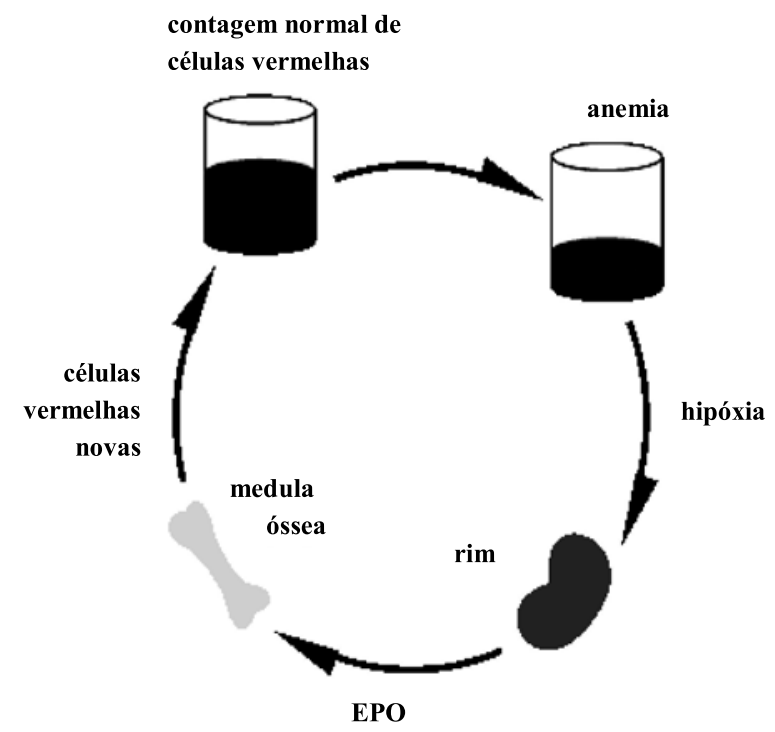

Fonte: Adaptado de: http://www.noblood.org/gallery/ image-erythropoietin-hypoxia-11.html

\section{Produtos biológicos}

Em 1972, surge o que conhecemos hoje como tecnologia do DNA recombinante (rDNA). De forma inédita, Berg utiliza enzimas de restrição para cortar e reunir pedaços de DNA de espécies diferentes (MATTE, 1999). Alguns anos depois, em 1977, a E. coli é utilizada para clonar, pela primeira vez, um hormônio humano, a somatostatina.

Em 1978, a Biotecnologia começa a dar seus frutos. Com a tecnologia do DNA recombinante, uma indústria de biotecnologia californiana clona e sintetiza, em bactérias manipuladas em laboratório, a insulina humana. Quatro anos depois, ela já era comercializada (BERG; MERTZ, 2010).

Desde então, o avanço técnico-científico tem permitido o emprego industrial de micro-organismos ou células geneticamente modificadas com vistas à produção de proteínas de interesse em diversas áreas, em especial, na saúde humana. Essa tecnologia permite reproduzir proteínas idênticas às naturais, bem como elaborar outras totalmente novas, mediante alterações correspondentes à inserção de genes nesses micro-organismos ou células. Tais moléculas podem se revelar mais vantajosas do que as naturais para uma determinada função, podendo, por exemplo, apresentar maior atividade biológica, meia-vida mais apropriada ao uso terapêutico ou efeitos colaterais mais brandos.

Desde 1980, esses produtos baseados na tecnologia do DNA recombinante têm sido produzidos e comercializados. No Brasil, as principais aplicações dessa tecnologia estão na produção de medicamentos biológicos, tais como vacinas, reagentes para diagnósticos e hemoderivados (SOARES, 2005). Os medicamentos biológicos, também chamados de biofármacos, produtos biológicos ou ainda biomedicamentos, podem ser definidos como aqueles obtidos de fluidos biológicos ou de tecidos de origem animal, e de procedimentos biotecnológicos (FARDELONE; BRANCHI, 2006; BRASIL, 2010a).

\section{Eritropoietina humana recombinante (rhEPO)}

Em 1977, a EPO foi extraída e purificada da urina de pacientes anêmicos. Utilizando-se da tecnologia do rDNA, baseada na sequência de aminoácidos dos dados 
da purificação, em 1983, o gene da EPO humana foi então clonado permitindo o desenvolvimento da rhEPO, um avanço tecnológico que revolucionou o tratamento da anemia.

A rhEPO é produzida em células de ovário de hamster chinês (CHO). Essa célula é amplamente utilizada, pois tem alta taxa de produção, é adequada para cultura em larga escala e a estrutura e composição das glicoproteínas são similares às dos seres humanos (WANG; SEMENZA, 1993), conferindo a rhEPO uma sequência de aminoácidos idêntica à da eritropoietina endógena (MIYAKE; KUNG; GOLDWASSER, 1977; JACOBS et al., 1985).

A rhEPO é uma glicoproteína composta de 165 aminoácidos que formam cadeia polipeptídica com duas pontes dissulfeto intramoleculares nas posições $\left(\mathrm{Cis}^{7-161}\right.$ e $\left.\mathrm{Cis}^{29-33}\right)$. Apresenta três sítios de glicosilação N-ligados $\left(\mathrm{Asn}^{24}, \mathrm{Asn}^{38} \mathrm{e} \mathrm{Asn}^{83}\right.$ ) que podem formar de duas a quatro cadeias sialiladas, e uma O-ligada $\left(\mathrm{Ser}^{126}\right)$ com até dois resíduos. Pode conter até 14 ácidos siálicos, necessários para que o hormônio atinja os sítios-alvo, evitando a rápida metabolização pelos receptores hepáticos que reconhecem as estruturas expostas de galactose, com posterior excreção. A massa molecular da estrutura glicosilada, que contém cerca de $40 \%$ de carboidratos, é de 30-34 kDa, e somente a da cadeia peptídica é de $18 \mathrm{kDa}$. A atividade específica deve ser maior ou igual a 100.000 UI/mg de proteína (SCHMIDT et al., 2003; COUNCIL OF EUROPE, 2011).

Assim como ocorre em outras proteínas glicosiladas, a rhEPO é encontrada como uma mistura de isoformas relacionadas com o mesmo padrão de glicosilação. Dados encontrados na literatura demonstram que o ponto isoelétrico varia de 4,5-6,5 e essa variação está relacionada com a heterogeneidade glicídica, principalmente com relação aos níveis de ácido siálico, que é um glicídio carregado. A maior parte das isoformas presentes na rhEPO encontra-se na faixa de ponto isoelétrico de 4,5-5,0 (FUKUDA et al., 1989; CONCEIÇÃO, 2003).

A avaliação do ponto isoelétrico, além de fornecer informações a respeito da verificação da manutenção das características físico-químicas da rhEPO presente nas formulações analisadas, possibilita também uma avaliação com relação aos níveis de ácido siálico da molécula. Segundo Fukuda et al. (1989), o ácido siálico está diretamente relacionado com a farmacocinética da rhEPO.
Estudos realizados pelo mesmo autor demonstraram que, quando os resíduos de ácido siálico terminais presentes nas cadeias oligossacarídicas da rhEPO são removidos, o tempo de meia-vida da proteína no plasma torna-se muito pequeno, impedindo assim que a EPO desempenhe seu papel na manutenção dos níveis de eritrócitos (CONCEIÇÃO, 2003).

As eritropoietinas alfa e beta são produzidas em células $\mathrm{CHO}$ - a célula de escolha para a produção da rhEPO - e têm as menores diferenças estruturais em relação ao padrão de glicosilação, mas os mesmos efeitos fisiológicos da EPO humana. A rhEPO produzida em células BHK (linhagem contínua de células de rim de feto de hamster; do inglês, Baby Hamster Kidney) - a omega epoetina - tem padrão de glicosilação diferente, assim como a Deltaepoetina, a qual é produzida em fibroblasto humano - HT1082 (WHO, 2010).

A primeira rhEPO comercializada nos Estados Unidos foi a Epogen (alfaepoetina da Amgen), em seguida, a Eprex (alfaepoetina da Ortho Biotech Products) e, posteriormente, na Europa, a NeoRecormon (betaepoetina da F.Hoffmann-LaRoche Ltd.).

A patente das primeiras eritropoietinas disponíveis comercialmente expirou, o que levou ao surgimento de eritropoietinas similares no mercado mundial, chamadas de biossimilares. Esse fato causa preocupação aos órgãos regulatórios, sobretudo no que diz respeito à eficácia e à segurança de tais produtos, uma vez que eles não são idênticos aos originais (WHO, 2010; BRINKS et al., 2011).

\section{Novas classes de rhEPOs}

Pesquisas vêm sendo realizadas, seguindo diferentes abordagens, com o objetivo de estender a meia-vida da rhEPO; dentre elas estão a adição de polietileno glicol (PEG), em processos conhecidos como peguilação, e de cadeias de carboidratos, conhecidas como hiperglicosilação.

A EPO endógena e a recombinante, ambas apresentam micro-heterogeneidade na estrutura de carboidratos com variação no conteúdo de ácido siálico. A importância do conteúdo de ácido siálico foi notada em experimentos com isoformas de rhEPO, revelando uma direta e positiva relação entre o conteúdo de ácido siálico e a potência in vivo (EGRIE; BROWNE, 2002). 
As moléculas com o conteúdo de ácido siálico aumentado tiveram a afinidade pelo EPO- $\mathrm{R}$ reduzida e a meia-vida aumentada, sugerindo que a maior meia-vida foi um determinante mais forte para a potência do que a afinidade pelo receptor. Consequentemente, a mistura de glicoformas foi importante para definir as propriedades biológicas de rhEPO. Essa observação também nos leva a pensar que a modificação da molécula de EPO, pela adição de mais cadeias de carboidratos e aumento do conteúdo de ácido siálico (>14 resíduos), pode prolongar a meia-vida sérica e elevar a potência quando comparada à rhEPO convencional (EGRIE; BROWNE, 2002; ELLIOTT; PHAM; MACDOUGALL, 2008 ).

A Darbepoetina alfa é um análogo hiperglicosilado que contém duas cadeias adicionais de carboidratos $\mathrm{N}$-ligados nas posições 30 e 88, como resultados de cinco substituições de aminoácidos (Ala30Asn, His32Thr, Pro87Val, Trp88Asn, Pro90Thr). Estudos mostram que essas substituições não interferiram diretamente na ligação com o receptor ou modificaram a estrutura e a estabilidade da molécula. A porção de carboidrato foi aumentada de $40 \%$ para $51 \%$, e o peso molecular aproximado elevado de $30 \mathrm{kDa}$ para $37 \mathrm{kDa}$. O número máximo de resíduos de ácido siálico aumentado de 14 para 22. Comparado com a alfaepoetina, a darbepoetina alfa teve a meia-vida três vezes mais longa, mais baixa afinidade de ligação com o receptor e melhor atividade in vivo em múltiplas espécies (EGRIE; BROWNE, 2001; EGRIE; BROWNE, 2002; ELLIOTT et al., 2003; ELLIOTT; PHAM; MACDOUGALL, 2008).

A adição de PEG - peguilação - em regiões reativas de proteínas ou carboidratos, tanto em solução ou em fase sólida, tem sido utilizada com sucesso para estender a meia-vida sérica de diferentes proteínas recombinantes. Moléculas peguiladas têm a hidrodinâmica aumentada. Essa hidrodinâmica aumentada pode resultar na redução da liberação devido à taxa reduzida de translocação do sangue para tecidos extravasculares. Tem-se considerado que PEG é relativamente inerte e não imunogênico, sendo assim um material adequado para conjugação de proteínas terapêuticas. Moléculas de rhEPO peguiladas, tipicamente contêm PEG ligado a diferentes aminas reativas, cada uma delas pode ter efeitos diferenciais na atividade e no envelopamento de proteínas (WANG et al., 2010).

A metoxipolietilenoglicol - betaepoetina (PEG EPO) da F. Hoffmann - La Roche Ltd., Basel, Suíça, foi aprovada, em 2007, nos Estados Unidos e na Europa, é um peguilado da betaepoetina. Parâmetros farmacocinéticos da PEG-EPO foram medidos em pacientes que receberam diálise peritoneal. A meia-vida média foi de 134 horas, quando administrada via intravenosa e, 139 horas, quando administrada por via subcutânea. A meia-vida longa dessa EPO permite sua administração uma vez por mês na fase de manutenção da terapia. (MACDOUGALL et al., 2006; ELLIOTT; PHAM; MACDOUGALL, 2008; PADULLÉ-ZAMORA et al., 2012).

Tanto a hiperglicosilação quanto a peguilação podem reduzir a afinidade de ligação com o receptor, embora a redução seja consideravelmente maior para rhEPO peguiladas do que para hiperglicosiladas ( 50 a 100 vezes e 5 vezes, respectivamente). A reduzida ligação com o EPO-R resulta em decréscimo, correspondentes, da potência in vitro dessas moléculas (ELLIOTT; PHAM; MACDOUGALL, 2008).

\section{Uso terapêutico da rhEPO}

O primeiro relato de uso clínico da rhEPO foi feito por Winearls et al. (1986) no Reino Unido e, posteriormente, por Eschbach et al. (1987) nos Estados Unidos, ainda durante estudos clínicos. Em 1989, a primeira rhEPO é aprovada nos EUA pelo FDA (Food and Drug Administration).

Antes do desenvolvimento da rhEPO, a transfusão de sangue foi o mais comum tratamento para pacientes com anemia, contudo, desde o seu advento, a rhEPO vem sendo amplamente utilizada na prática clínica, para redução da necessidade de transfusão sanguínea em processos cirúrgicos, para aumentar os níveis de hemoglobina (FARIS; RITTER; ABELS, 1996) e no tratamento de anemias de várias etiologias, incluindo anemia devido à doença renal crônica, anemia relacionada ao câncer ou ao tratamento dele (RIZZO et al., 2001; ELLIOTT; PHAM; MACDOUGALL, 2008), anemia relacionada ao uso de zidovudina, em pacientes infectados pelo HIV (SAAG; LEVINE; LEITZ, 2002) e anemia relacionada à utilização de ribavirina, em pacientes portadores de hepatite $\mathrm{C}$ (SOLKOWSKY; WASSERMAN; BRAU, 2001).

A rhEPO também está indicada para pacientes oncológicos com malignidades de origem não mieloide, cuja anemia se deva diretamente ao efeito concomitante de 
quimioterapia administrada por, no mínimo, dois meses. A rhEPO está indicada nesse grupo de pacientes para elevar ou manter o nível do hematócrito ou de hemoglobina e, assim, reduzir o número de transfusões sanguíneas, bem como melhorar a resposta à terapia. Não está indicada para os casos de anemia grave que requeiram correção imediata. A rhEPO substitui a necessidade de tratamento com transfusão, mas não a transfusão de emergência em qualquer que seja o caso. Também não é indicada para a correção da anemia, nesse grupo particular de pacientes, quando ela for devida a outros fatores como a deficiência de folato, hemólise ou sangramento gastrointestinal (FRIED, 2009; BIO-MANGUINHOS, 2011).

Um fator de grande relevância, quando do uso da rhEPO, está na melhora da qualidade de vida e, de maneira geral, na sobrevida, ocorrendo um decréscimo na sensação de fadiga. $O$ uso da rhEPO pelos pacientes permite normalizar o seu apetite e acelerar o seu retorno às atividades de rotina (AAPRO et al., 2006; GUAN; CHEN, 2008).

\section{A rhEPO além do tratamento da anemia}

Existem estudos que avaliaram o uso de rhEPO em outras etiologias, sugerindo a interação da rhEPO com seus receptores, os quais têm sido identificados em células tumorais (FANDRY, 2008), e ainda que a rhEPO possa reagir com receptores em células endoteliais para estimular a angiogênese (RIBATTI et al., 2007), sugerindo funções não eritroides para a EPO.

Uma pesquisa mostrou que o uso da rhEPO melhora o sistema imune por estar associada com efeitos em uma variedade de parâmetros e funções imunológicas, sugerindo também uma função antimieloma. (PRUTCHI-SAGIV et al., 2006).

Algumas investigações mostraram que a administração de rhEPO antes ou logo depois do início da isquemia, reduziu o tamanho do infarto no miocárdio (IM) e melhorou a função cardíaca em fases agudas (CALVILLO et al., 2003; HIRATA et al., 2005; MINAMINO; KITAKAZE, 2006).

Recentemente, Hirata et al (2005) demonstraram em modelo animal que a rhEPO aumenta a formação de células progenitoras endoteliais assim como a angiogênese em miocárdio isquêmico após o IM, enquanto melhora a função cardíaca, sugerindo ainda que alguns potenciais mecanismos, pelos quais a EPO pode induzir a cardioproteção, seriam a redução da morte celular por apoptose; a angiogênese (pode contribuir para melhora da função cardíaca após o IM); e a prevenção do remodelamento em áreas não infartadas.

O potencial papel protetor da rhEPO, em modelos animais para infarto do miocárdio, tem sido avaliado e os efeitos benéficos têm sido observados independentemente de a administração ter sido feita antes da isquemia, no início da isquemia ou na reperfusão (PATEL et al., 2011). Isso sugere que o tratamento com a EPO é efetivo para a prevenção e tratamento da isquemia.

A EPO é conhecida por ser a maior reguladora da formação vascular (i.e angiogênese), do crescimento de órgãos no embrião e o EPO-R tem sido encontrado em quase todos os tecidos embrionários. A rhEPO estimula a sobrevivência e a proliferação de células endoteliais in $v i$ tro e promove a formação de novos vasos sanguíneos. No modelo murino, a EPO estimulou a angiogênese na cicatrização de feridas (RIBATTI et al., 2007; RIBATTI, 2008).

O primeiro relato do efeito neuroprotetor da rhEPO, administrada por uma via periférica, foi dado por Brines et al (2000) que demonstraram, em um modelo de acidente vascular cerebral focal, uma significante redução do volume do infarto quando da aplicação de uma alta dose (5000 UI/kg) de rhEPO injetada por via intraperitonial até seis horas após a reperfusão.

A EPO e seu receptor estão presentes no sistema nervoso central, incluindo a retina. In vitro, a EPO protegeu os neurônios contra a isquemia induzida pela toxicidade ao glutamato, o primeiro fator responsável pela morte de células neuronais relacionadas à hipóxia. (BUEMI et al., 2003; WEISS, 2003).

A rhEPO tem demonstrado ter efeito antioxidante. O exato mecanismo não está completamente elucidado, porém várias hipóteses têm sido sugeridas. Uma delas é que a EPO apresenta ação antioxidante direta pela remoção de radicais livres (YANG et al., 2011).

\section{Riscos associados ao uso de rhEPO}

Os principais efeitos colaterais relacionados ao uso da rhEPO são: aumento da hipertensão arterial préexistente, crises convulsivas, hiperemia conjuntival, even- 
tos trombóticos e cefaleia (BIO-MANGUINHOS, 2011; ABENSUR, 2000).

A expressão da EPO e de seu receptor tem sido reconhecida em uma variedade de cânceres humanos, incluindo de mama, próstata, colo, ovário, útero, glioblastoma e carcinoma de células escamosas de cabeça e pescoço. Testes in vitro e em modelos animais têm mostrado que o EPO-R pode estar presente em uma gama de tumores e linhagens de células cancerosas (HARDEE et al., 2006; OSTERBORG et al., 2007).

A EPO não somente estimula a proliferação de células cancerosas e inibe a apoptose, mas também estimula a migração e invasão dessas células. $\mathrm{O}$ completo entendimento da função do EPO-R em células tumorais ainda permanece ausente na literatura (HARDEE et al., 2006; GUAN; CHEN, 2008).

Outro ponto que merece atenção é o risco de trombose. De acordo com estudo baseado na revisão sistemática e triagem de 9.353 pacientes e outro que avaliou o uso de uma alta dose de EPO (40.000 - 60.000 UI por semana), administrada por via subcutânea, ambos em pacientes com câncer, demonstraram que o tratamento com epoetina ou darbepoetina aumentou o risco e a incidência de eventos tromboembólicos (BOHLIUS et al., 2006; MINAMINO; KITAKAZE, 2006).

Apesar dos benefícios do uso da EPO, que são de grande importância, é preciso cautela quanto à sua aplicação clínica. Isso mostra a importância de se seguir as indicações autorizadas do uso da rhEPO.

\section{Controle da qualidade da rhEPO}

Diante da gama de produtos disponíveis no mercado, da abrangência da indicação terapêutica e das características dos pacientes usuários de rhEPO, é de grande importância o efetivo controle da qualidade desse produto previamente ao seu ingresso no mercado. $\mathrm{O} \mathrm{Mi}$ nistério da Saúde, em 2005, passou a fornecer produtos biológicos, dentre eles a rhEPO, por meio de acordo entre Brasil e Cuba, ficando a Fiocruz na liderança do grupo nacional de detentores da tecnologia para a produção de biofármacos (FARDELONE; BRANCHI, 2006).

Desde 1983, o INCQS faz o controle da qualidade de produtos biológicos, entre eles soros hiperimunes e vacinas, utilizados no Programa Nacional de Imunizações (PNI), sendo igualmente responsável pelo controle da qualidade de alguns biofármacos, i.e. rhEPO e Interferon alfa $2 \mathrm{~b}$ humano recombinante, que são adquiridos pelo MS.

Dentre os ensaios preconizados nos compêndios oficiais e realizados pelo INCQS para o controle da qualidade da rhEPO (Tabela 1), estão: (i.) análise de protocolo resumido de produção e controle (PRPC); (ii.) avaliação da potência biológica; (iii.) determinação de $\mathrm{pH}$; (iv.) volume médio; (v.) identificação por imunoblot; (vi.) identificação por SDS-PAGE; (vii.) esterilidade; e (viii.) endotoxina bacteriana.

Atualmente não existe monografia para rhEPO na Farmacopeia Brasileira; dessa forma, o compendio adotado como referência no Brasil é a Farmacopeia Europeia (Ph. Eur.).

Tabela 1 - Ensaios realizados no INCQS para o controle da qualidade da rhEPO

\begin{tabular}{|c|c|c|}
\hline ENSAIO & VALOR DE REFERÊNCIA & FONTE \\
\hline Análise de protocolo & De acordo com a norma oficial & $\begin{array}{c}\text { Farmacopeia Europeia, } \\
7.0-2011\end{array}$ \\
\hline Potência biológica & $\begin{array}{c}\text { Entre } 80 \text { a } 125 \% \text { e limites de confiança } \\
\text { entre } 64 \text { e } 156 \% \text { do rotulado }\end{array}$ & $\begin{array}{c}\text { Farmacopeia Europeia, } \\
7.0-2011 \\
\end{array}$ \\
\hline Determinação de pH & 6,5 a 7,5 & Registro do produto \\
\hline Volume médio & Mínimo de 1,1 mL/frasco & $\begin{array}{l}\text { Farmacopeia Brasileira, } \\
\text { 4a edicão, parte I, } 1998\end{array}$ \\
\hline Identificação por Imunoblot & Reativo com reagente & $\begin{array}{c}\text { Farmacopeia Europeia, } \\
\text { 7.0, } 2011\end{array}$ \\
\hline Identificação por SDS-PAGE & $\begin{array}{c}\text { Conforme a faixa de peso molecular } \\
\text { esperada }\end{array}$ & $\begin{array}{c}\text { Farmacopeia Europeia, } \\
\text { 7.0, } 2011\end{array}$ \\
\hline Esterilidade & $\begin{array}{l}\text { Ausência de bactérias e fungos } \\
\text { contaminantes }\end{array}$ & $\begin{array}{l}\text { Farmacopeia Brasileira, } \\
\text { 4ª edição, parte I, } 1998\end{array}$ \\
\hline Endotoxina bacteriana & $\begin{array}{c}<20 \text { IU para um volume que contenha } \\
100.000 \text { IU de eritropoietina }\end{array}$ & $\begin{array}{c}\text { Farmacopeia Europeia, } \\
\text { 7.0, } 2011\end{array}$ \\
\hline
\end{tabular}




\subsection{Avaliação da potência biológica para rhEPO}

A atividade biológica, ou potência biológica, é definida como a habilidade específica ou capacidade do produto atingir um efeito biológico definido (BRASIL, 2010a), no caso da rhEPO, a correção da anemia, i.e. a elevação do nível de hemoglobina e hematócrito. Essa é uma avaliação laboratorial de suma importância, uma vez que seu resultado está relacionado à eficácia do produto final. Assim, a avaliação da potência biológica é um dos ensaios preconizados pela Farmacopeia Europeia ( $P h$. Eur.) em produtos contendo rhEPO a fim de que possam ser liberados para uso da população.

Os primeiros bioensaios in vivo preconizados para avaliação da potência biológica da rhEPO foram baseados na captação de $\mathrm{Fe}^{59}$ radioativo. (METCALF, 2008). Atualmente, essa avaliação pode ser realizada por dois métodos. Um método (método A) utiliza camundongos policitêmicos e outro (método B) utiliza camundongos normocitêmicos (COUNCIL OF EUROPE, 2011). O método B tem sido o método de escolha, uma vez que o método $\mathrm{A}$ - em camundongos policitêmicos - causa maior sofrimento e estresse aos animais, que devem ser mantidos por longos períodos em câmaras hipobáricas e expostos a radioisótopos (ECVAM, 2002). Como resultado dessa escolha, diferentes linhagens de camundongos normocitêmicos têm sido testadas para a avaliação da potência de rhEPO, tais como CF1, Balb/c, e B6D2F1 (ALBERTENGO et al., 1999; RAMOS et al., 2003; SCHMIDT et al., 2003; BARTH et al., 2008; COUNCIL OF EUROPE, 2011).

Esse método tem como base a medida do estímulo da produção de reticulócitos após a injeção de rhEPO, sem qualquer exposição prévia.

O ensaio é realizado com desenho de blocos ao acaso, no qual os animais são distribuídos de forma randômica. O tratamento é feito por via subcutânea em que alguns animais são inoculados com a amostra teste e outros com padrão de referência, sendo seis representantes de cada dose, totalizando 36 animais em cada teste.

Após 96 horas (para a linhagem B6D2F1), é realizada a coleta de sangue por via plexo orbital, seguida do processamento do sangue que consta da coloração do material genético dos reticulócitos com corante vital azul de metileno e da indução da hemólise seletiva. Posteriormente, tais células são contadas em hemocitômetro (câmara de Neubauer).
Um único trabalho experimental testou o uso da linhagem Swiss Webster (SW), sugerindo sua aplicação na avaliação da rhEPO como alternativa válida à linhagem B6D2F1, preconizada pela Ph. Eur. (LOPES, 2004). No entanto, por não ter seguido compêndio oficial e por não ter avaliado a correlação entre essa linhagem alternativa e a linhagem preconizada oficialmente - B6D2F1 - a linhagem SW não pôde até hoje ser implantada para fins regulatórios.

Nesse contexto, ensaios preliminares para avaliação da potência da rhEPO foram realizados, utilizando a linhagem SW. Com base nos resultados desses ensaios, publicados por nosso grupo (COSTA et al., 2010), foi possível ajustar algumas variáveis como faixa ideal de peso corpóreo dos animais, melhor esquema de doses, melhor dia para contagem de reticulócitos etc. que deverão nortear a validação do uso da linhagem SW para avaliar a potência de rhEPO.

\section{Situação da rhEPO no Brasil}

O tratamento padrão para a anemia na doença renal crônica, atualmente, consiste na administração de agentes estimulantes da eritropoiese. Dentre eles, por ter uma melhor relação custo-benefício, a rhEPO é o fármaco mais utilizado na terapêutica, inclusive no Brasil (GURGEL et al., 2012).

A rhEPO é um medicamento que faz parte do Componente Especializado de Assistência Farmacêutica, aprovado pela Portaria no 2981 de 26/11/2009 do Ministério da Saúde (BRASIL, 2009), como parte da Política Nacional de Assistência Farmacêutica. Para atender ao exposto nessa Portaria, o MS, em parceria com a Secretaria de Ciência e Tecnologia e Insumos Estratégicos (SCTIE), financia o medicamento em cofinanciamento com as Secretarias Estaduais de Saúde, que também são responsáveis pela aquisição, distribuição e dispensação do medicamento ao usuário final, para garantir à população acesso gratuito a rhEPO.

Avaliando os protocolos clínicos e diretrizes terapêuticas, elaborados pelo MS, a rhEPO está indicada e tem sido prescrita para uso ambulatorial e hospitalar, para casos de anemia na insuficiência renal crônica; hepatite $\mathrm{C}$ e transplante de intestino, medula ou pâncreas (BRASIL, 2010b). 
Em consulta realizada no site da Agência Nacional de Vigilância Sanitária (Anvisa) em 20 de março de 2012, observou-se a existência de quatro produtos registrados, de quatro diferentes empresas, quando o termo busca foi "princípio ativo: eritropoetina humana recombinante". Quatro produtos de quatro diferentes empresas, quando o termo busca foi "princípio ativo: alfaepoetina" e dois produtos de uma mesma empresa, quando o termo busca foi "princípio ativo: betaepoetina". Essa consulta mostrou que temos um número considerável de diferentes empresas (oito) que detêm tecnologia para a produção de rhEPO, levando a uma gama de diferentes produtos (10). E ainda com diferentes países fabricantes (oito), com produtos registrados no Brasil. Todas as buscas consideraram registros válidos, ou seja, dentro do prazo de vigência.

O INCQS recebe anualmente cerca de 130 lotes rhEPO para análise, o que corresponde à demanda do Ministério da Saúde, por meio da Secretaria de Ciência, Tecnologia e Insumos Estratégicos (SCTIE). Ainda assim, esse quantitativo de lotes não consegue atender plenamente aos pacientes, a maioria deles renais crônicos, que recebem a rhEPO por meio do SUS (Sistema Único de Saúde).

A Secretaria de Vigilância Sanitária publicou em seu clipping que, no Pará, segundo a Associação dos Renais Crônicos e Transplantados do Estado (ARCT-PA), os pacientes estariam com dificuldades para obter o medicamento eritropoietina. Segundo a ARCT-PA, o Ministério da Saúde repassa 10 mil frascos com 4000 mg cada; no entanto, existem 1.728 pacientes renais crônicos cadastrados que dependem da rhEPO e cada um recebe por mês 12 frascos de rhEPO, com 4000 mg cada, ou seja, um quantitativo insuficiente para a demanda do Pará (BRASIL, 2011b).

Outro exemplo de desabastecimento ocorreu em Sumaré - São Paulo, onde pacientes protestaram contra a falta de rhEPO nas farmácias do SUS. A falta desse medicamento, como relatam os pacientes, pode agravar a situação de indivíduos que sofrem hemodiálise, uma vez que ele ajuda a manter o quadro de saúde (BRASIL, 2011a).

\subsection{Lotes analisados pelo INCQS}

No INCQS, a avaliação da qualidade de lotes de rhEPO utilizados pelo MS teve início no ano de 2007. Com base nos dados oriundos dos Sistemas de Gerenciamento de Amostras (SGA-web e SGA 2000) utilizados no
INCQS e do Protocolo Resumido de Produção e Controle - PRPC (Tabela 2), foi possível observar que, no primeiro ano de análise, foram recebidos 96 lotes. Nos anos seguintes, de 2008 a 2012 (até o mês de junho de 2012), foram recebidos 136, 150, 144, 170 e 83 lotes, respectivamente, totalizando 779 lotes. Esse total de lotes corresponde a aproximadamente 6,5 milhões de frascos de rhEPO liberados anualmente para consumo.

Considerando a forma de apresentação (2000 UI ou $4000 \mathrm{UI} / \mathrm{frasco}$ ), a maioria dos lotes recebidos no período de 2007 a 2012 foi de 4000 UI/frasco. A apresentação de $2000 \mathrm{UI} /$ frasco consistiu cerca de 10\% do total de lotes recebidos em todo o período de estudo.

Tabela 2 - Quantitativo de lotes recebidos no INCQS

\begin{tabular}{c|c|c|c|c}
\hline ANO & $\begin{array}{c}\text { TOTAL DE } \\
\text { LOTES }\end{array}$ & $\mathbf{2 0 0 0}$ UI & $\mathbf{4 0 0 0}$ UI & I (\%) \\
\hline 2007 & 96 & $27(28 \%)$ & $69(72 \%)$ & 0 \\
\hline 2008 & 136 & $6(4 \%)$ & $130(96 \%)$ & 0 \\
\hline 2009 & 150 & $16(11 \%)$ & $134(89 \%)$ & 0 \\
\hline 2010 & 144 & $19(13 \%)$ & $125(87 \%)$ & 0 \\
\hline 2011 & 170 & $18(11 \%)$ & $152(89 \%)$ & 0 \\
\hline $2012^{\#}$ & 83 & $7(8 \%)$ & $76(92 \%)$ & $4^{*}$ \\
\hline Soma & $\mathbf{7 7 9}$ & $\mathbf{9 3 ( 1 2 \% )}$ & $\mathbf{6 8 6 ( 8 8 \% )}$ & $\mathbf{4}$ \\
\hline
\end{tabular}

\# Dados referentes ao período de janeiro a junho de 2012; (\%) percentual em relação ao total daquele ano. Fonte: INCQS (2012).

\section{Considerações finais}

Milhões de pacientes em todo mundo são beneficiados com o uso da rhEPO, a maioria deles com doença renal crônica, o que tem um impacto importante na saúde pública, sobretudo devido à redução da necessidade de transfusões de sangue e à melhor qualidade de vida dos pacientes.

Dentre os medicamentos de alto custo disponibilizados pelo SUS, a rhEPO tem sido o mais utilizado. Esse é o medicamento de escolha para pacientes em hemodiálise, em virtude da maior severidade da anemia nesses pacientes e seu menor custo em relação aos outros medicamentos estimuladores da eritropoiese.

Diante da crescente demanda no cenário nacional, do alcance de sua indicação terapêutica e das características dos pacientes usuários de rhEPO, é de grande importância o efetivo controle da qualidade desse produto, garantindo assim segurança e eficácia na utilização desse medicamento biológico. 


\section{Referências}

AAPRO, M. S. et. al. Epoetin alfa increases hemoglobin levels and improves quality of life in anemic geriatric cancer patients receiving chemotherapy. Supportive Care Cancer, Heidelberg, v. 14, n. 12, p. 1184-1194, dec. 2006. doi: $10.1007 / \mathrm{s} 00520-006-0076-\mathrm{Z}$

ABENSUR, H. Reações adversas associadas à eritropoietina. Jornal Brasileiro de Nefrologia, São Paulo, v. 22, supl. 5, p. 39-41, jan./mar. 2000.

ALBERTENGO, M. E. et al. Eritropoyetina recombinante humana: método de valoración in vivo con ratones normocitémicos. Sangre, Barcelona, v. 44, n. 5, p. 357-363, oct. 1999.

BARTH, T. et al. Validation of the normocythemic mice bioassay for the potency evaluation of recombinant human erythropoietin in pharmaceutical formulations. Journal of AOAC International, Arlington, v. 91, n. 2, p. 285-291, mar. 2008.

BERG, P.; MERTZ, J. E. Personal reflections on the origins and emergence of recombinant DNA technology. Genetics, Bethesda, v. 184, n. 1, p. 9-17, jan. 2010. doi: 10.1534/genetics.109.112144

\section{BIO-MANGUINHOS/FIOCRUZ.}

Eritropoetina

humana recombinante: monografia do produto. Disponível em: $<$ http://www.bio.fiocruz.br/images/stories/ pdfs/monografia_epo.pdf $>$. Acesso em: 31 maio 2011.

BOHLIUS, J. et al. Recombinant human erythropoietins and cancer patients: updated meta-analysis of 57 studies including 9353 patients. Jounal of National Cancer Institute, Oxford, v. 98, n. 10, p. 708-714, may. 2006. doi: $10.1093 /$ jnci/djj189

BRASIL. Ministério da Saúde. Gabinete do Ministro. Portaria no 2981 de 26 de novembro de 2009. Aprova o Componente Especializado da Assistência Farmacêutica. Diário Oficial [da] República Federativa do Brasil, Brasília, DF, 30 de novembro de 2009, Seção 1, n. 228, p. 725-771.

BRASIL. Agência Nacional de Vigilância Sanitária. RDC No. 55, de 16 de dezembro de 2010. Dispõe sobre o Registro de Produtos Biológicos novos e produtos biológicos e dá outras providências. Diário Oficial [da] República Federativa do Brasil, Brasília, DF, 17 de dezembro de 2010a. Seção 1, n. 241, p. 110-113.

BRASIL. Ministério da Saúde. Secretaria de Ciência, Tecnologia e Insumos Estratégicos. Departamento de Assistência Farmacêutica e Insumos Estratégicos. Da excepcionalidade às linhas de cuidado: $\mathrm{O}$ Componente Especializado da Assistência Farmacêutica. Série B. Textos Básicos de Saúde, Brasília, DF, 2010b. 262 p. il..
BRASIL. Ministério da Saúde. SVS - Secretaria de Vigilância Sanitária. Falta de medicamentos gera protestos de moradores. Clipping SVS. Brasília, DF, janeiro, 2011a. Disponível em: <http://portal.saude. gov. br/portal/arquivos/pdf/clipping_31012011.pdf $>$. Acesso em: 28 mar. 2012.

BRASIL. Ministério da Saúde. SVS - Secretaria de Vigilância Sanitária. Doentes sofrem sem remédios. Clipping SVS. Brasília, DF, maio, 2011b. Disponível em: $<$ http://portal.saude.gov.br/portal/arquivos/pdf/clippi ng_24052011.pdf $>$. Acesso em: 28 mar. 2012.

BRINES, M. L. et al. Erythropoietin crosses the bloodbrain barrier to protect against experimental brain injury. Proceedings of the National Academy of Science, Washington, v. 97, n. 19, p. 10526-10531, sep. 2000. doi: 10.1073/pnas.97.19.10526

BRINKS, V. et al. Quality original and biosimilar epoetin Products. Pharmaceutical Research, Stuttgart, v. 28, n. 2, p. 386-393, feb. 2011. doi: 10.1007/s11095-010-0288-2

BUEMI, M. et al. The pleitropic effects of erythropoietin in the central nervous system. Jounal of Neuropathology \& Experimental Neurology, Philadelphia, v. 62, n. 3, p. 228-236, mar. 2003.

CALVILLO, L. et al. Recombinant human erythropoietin protects the myocardium from ischemia reperfusion injury and promotes beneficial remodeling. Proceedings of the National Academy of Science, Washington, v. 100 , n. 8 , p. $4802-4806$, apr. 2003. doi: 10.1073/ pnas. 0630444100

CONCEIÇÃO, C. M. Isolamento e caracterização físico-química e química parcial de eritropoietina humana recombinante de formulações terapêuticas. 2003. 175p. il. Dissertação (mestrado) - Instituto de Química, Universidade Federal do Rio de Janeiro, Rio de Janeiro, 2003.

COSTA, R. N. et al. Evaluation of the applicability of Swiss Webster lineage on the biological potency test of recombinant human rrythropoietin. International Journal Biosafety and Biosecurity, Rio de Janeiro, v. 1, n. 1, p. 49-59, nov. 2010.

COUNCIL OF EUROPE. Erythropoietin concentrated solution. In: The European Pharmacopoeia, Monograph 1316, $7^{\text {th }}$ edition, v. 1, Strasbourg, 2011, p.1813-1817.

ECVAM - European Centre for the Validation of Alternative Methods. Scientific Advisory Committee. Statement on the batch potency testing of erythropoietin concentrated solution. Atla, Nottingham, v. 30, n. 5, p. 487-489, sep. 2002. 
EGRIE, J.; BROWNE, J. Development and characterization of novel erythropoiesis stimulating protein (NESP). British Journal of Cancer, London, v. 84, supl. 1, p. 3-10, apr. 2001. doi: 10.1054/bjoc.2001.1746

EGRIE, J.; BROWNE, J. Darbepoetina alfa is more potent in vivo and can be administered less frequently than rHuEPO. British Journal of Cancer, London, v. 87, n. 4, p. 476-477, aug., 2002. doi:10.1038/sj.bjc.6600506

ELLIOTT, S.; PHAM, E.; MACDOUGALL, I. C. Erythropoietins: A common mechanism of action. Experimental Hematology, Philadelphia, v. 36, n. 12, p. 1573-1584, dec. 2008. doi: 10.1016/j.exphem.2008.08.003.

ESCHBACH, J. W. et al. Correction of the anemia of end stage renal failure with recombinant human erythropoietin. Results of a combined phase I and II clinical trial. New England Journal of Medicine, Waltham, v. 316, n. 2, p. 7378, jan. 1987. doi: 10.1056/NEJM198701083160203

FANDRY, J. Erythropoietin receptors on tumor cells: what do they mean. The Oncologist, Durham, v. 13, suppl. 3, p. 16-20, jan. 2008. doi: 10.1634/theoncologist.13-S3-16

FARDELONE, L. C; BRANCHI, B. A. O setor de biofármacos e as oportunidades para o Brasil. Revista da FAE, Curitiba, v. 9, n. 2, p. 29-38, out. 2006.

FARIS, P. M; RITTER, M. A; ABELS, R. I. The effects of recombinant human erythropoietin on perioperative transfusion requirements in patients having major orthopedic operation. The American Erythropoietin Study Group. Journal of Bone and Joint Surgery, Boston, v. 78, n. 1, p. 62-72, jan. 1996.

FISHER, J. W. et al. Erythropoietin production by interstitial cells of hypoxic monkey kidneys. British Journal of Haematology, Oxford, v. 95, n. 1, p. 27-32, oct. 1996. doi: 10.1046/j.1365-2141.1996.d01-1864.x

FRIED, W. The liver as a source of extrarenal erythropoietin production. Blood, Washington, v. 40, n. 5, p. 671-677, nov. 1972.

FRIED, W. Erythropoietin and erythropoiesis. Experimental Hematology. Netherlands, v. 37, n. 9, p. 1007-1015, sep. 2009. doi: 10.1016/j.exphem.2009.05.010

FUKUDA, M. N. et al. Survival of recombinant erythropoietin in the circulation: the role of carbohydrates. Blood, Washington, v. 73, n. 1, p. 84-89, jan. 1989.

GILD, D. et al. Analytical methods for characterization and quality control of pharmaceutical peptides and proteins, using erythropoietin as an example. Pharmaceutica Acta Helvetiae, Zürich, v. 71 , n. 6, p. 383-394, dec. 1996. doi: 10.1016/S0031-6865(96)00048-9
GUAN, X; CHEN, L. Role of erythropoietin in cancerrelated anaemia: a double-edged sword? The Journal of International Medical Research, London, v. 36, n. 1, p. 1-8, feb. 2008. doi: 10.1177/147323000803600101

GURGEL, T. C. et al. Utilização de eritropoetina por pacientes incidentes em hemodiálise no Sistema Único de Saúde, Brasil, 2002-2003. Cadernos de Saúde Pública, Rio de Janeiro, v. 28 , n. 5, p. $856-868$, mai. 2012. doi: $10.1590 /$ S0102-311X2012000500005

HARDEE, M. E. et al. Erythropoietin biology in cancer. Clinical Cancer Research, Denville, v. 12, n. 2, p. 332-339, jan. 2006. doi: 10.1158/1078-0432.CCR-05-1771

HIRATA, A. et al. Erythropoietin just before reperfusion reduces both lethal arrhythmias and infarct size via the phosphatidylinositol-3 kinase-dependent pathway in canine hearts. Cardiovascular Drugs and Therapy, Norwell, v. 19, n. 1, p. 33-40, jan. 2005. doi: 10.1007/ s10557-005-6895-1

INCQS (Instituto Nacional de Controle de Qualidade em Saúde). Sistema de Gerenciamento de Amostras - SGA web e SGA 2000. Disponibilidade restrita. Acesso em: 03 jun. 2012.

JACOBS, $\mathrm{K}$. et al. Isolation and characterization of genomic and cDNA clones of human erythropoietin. Nature, London, v. 313, n. 6005, p. 806-810, mar. 1985. doi: $10.1038 / 313806 \mathrm{a} 0$

JACOBSON, L. O. et al. Role of the kidney in erythropoiesis. Nature, London, v. 179, n. 4560, p. 633-634, mar. 1957. doi:10.1038/179633a0

KAUSHANSKY, K; KIPPS, T. Hematopoietic agents: growth Factors, minerals and vitamins. In: BRUNTON, L. L.; LAZO, J. S.; PARKER, K. L. Goodman and Gilman's The Pharmacological Basis of Therapeutics. 11. ed. New York: McGraw-Hill, 2005. p. 1433-1465.

KOURY, S. T. et al. The use of in situ hybridization to study erythropoietin gene expression in murine kidney and liver. Microscopy Research and Technique, New York, v. 25, n.1 p. 29-29, may 1993. doi: 10.1002/jemt.1070250106

LLIOTT, S. et al. Enhancement of therapeutic protein in vivo activities through glycoengineering. Nature Biotechnology, New York, v. 21, n.4, p. 414-421, mar. 2003. doi:10.1038/nbt799

LOPES, M. C. Avaliação da potência biológica da eritropoetina humana recombinante em produtos farmacêuticos: estudo comparativo entre as linhagens de camundongos B6D2F1 e Swiss Webster. 2004. 73f. : il. Dissertação (mestrado). Instituto Nacional de Controle de Qualidade em Saúde, Fundação Oswaldo Cruz. 
MACDOUGALL, I. C. et. al. Pharmacokinetics and pharmacodynamics of intravenous and subcutaneous continuous erythropoietin receptor activator (C.E.R.A.) in patients with chronic kidney disease. Clinical Journal of the American Society of Nephrology, Washington, v. 1, n. 6, p. 1211-1215, nov. 2006. doi: 10.2215/ CJN.00730306

MATTE, U. Histórico de Fatos Relevantes em Genética 1859-1999. Texto incluído em 21/07/1999. Disponível em: $<$ http://www.ufrgs.br/bioetica/crogen.htm $>$. Acesso em: 25 maio 2011.

METCALF, D. Hematopoietic cytokines. Blood, Washington, v. 111, n. 2, p. 485-491, jan. 2008.

MINAMINO, T.; KITAKAZE, M. New therapeutic application of erythropoietin against ischemic heart diseases. Journal of Pharmacological Sciences, Kyoto, v. 101, n.2, p. 179 -181, jun., 2006.

MIYAKE, T.; KUNG, C. K-H.; GOLDWASSER, E. Purification of human erythropoietin. The Journal of Biological Chemistry, Baltimore, v. 252, n. 15, p. 55585564, aug. 1977.

OSTERBORG, A. et al. Preclinical studies of erythropoietin receptor expression in tumor cells: Impact on clinical use of erythropoietic proteins to correct cancer-related anemia. European Journal of Cancer, Amsterdam, v. 43, n. 3, p. 510-519, feb. 2007. doi: 10.1016/j.ejca.2006.10.012

PADULLÉS-ZAMORA, $\mathrm{N}$. et al. Use of methoxy polyethylene glycol-epoetin beta in stage 3, 4 or 5 nondialysis chronic kidney disease. Revista Nefrología, Barcelona, v. 32, n. 2, p. 221-227, jan. 2012. doi. 10.3265/ Nefrologia.pre2012.Jan.10946

PATEL, N. S. A. et al. Erythropoietin in the intensive care unit: beyond treatment of anemia. Annals of Intensive Care, Heidelberg, v. 1, n. 40, sep., 2011. doi: 10.1186/21105820-1-40

PLZAK, L. F. et al. Demonstration of stimulation of erythropoiesis by plasma from anemic rats using Fe59. Journal of Laboratory and Clinical Medicine, Amsterdam, v. 46, n. 1, p. 671- 678, jan. 1955.

PRUTCHI-SAGIV, S. et al. Erythropoietin treatment in advanced multiple myeloma is associated with improved immunological functions: could it be beneficial in early disease? British Journal of Haematology, Oxford, v. 135 , n. 5, p. 660-672, dec. 2006. doi: 10.1111/j.13652141.2006.06366.x

RAMOS, A. S. et al. Biological evaluation of recombinant human erythopoietin in pharmaceutical products. Brazilian Journal of Medical and Biological Research,
Ribeirão Preto, v. 36, n. 11, p. 1561-1569, nov. 2003. doi: 10.1590/S0100-879X2003001100014

RIBATTI, D. et al. Erythropoietin/erythropoietin receptor system is involved in angiogenesis in human hepatocellular carcinoma. Histopathology, Oxford, v. 50, n.5, p. 591-596, apr., 2007. doi: 10.1111/j.1365-2559.2007.02654.x

RIBATTI, D. Erythropoietin, the first century. Leukemia Research, Oxford, v. 32, n. 8, p. 1169-1172, mar. 2008. doi: 10.1016/j.leukres.2008.01.018

RIZZO, J. D. et al. Erythropoietin: a paradigm for the development of practice guidelines. Hematology/ The Education Program of the American Society of Hematology, Washington, v. 2001, n. 1, p. 10-30, jan. 2001. doi:10.1182/asheducation-2001.1.10

SAAG, M. S. et al. Once-weekly epoetin alfa improves quality of life and increases hemoglobin in anemic HIV+ patients. AIDS Research and Human Retroviruses, New York, v. 20 , n. 10, p. 1037-1045, oct. 2004. doi: 10.1089/ aid.2004.20.1037

SCHMIDT, C. A. et al. Avaliação da atividade e caracterização de Eritropoietina humana recombinante em produtos farmacêuticos. Arquivos Brasileiros de Endocrinologia eMetabologia, São Paulo, v. 47, n. 2, p. 183189, abr. 2003. doi: 10.1590/S0004-27302003000200012

SOARES, E. E. O Setor de biotecnologia. Mesa redonda: bionegócios. In: SIMPÓSIO NACIONAL DE BIOPROCESSOS, 15., 2005, Recife. Anais... Recife: UFPE, 2005.

SOLKOWSKY, M.; WASSERMAN, R.; BRAU, N. Once Weekly recombinant human erythropoietin (epoetin alfa) facilitates optimal ribavarin (RBV) doping in hepatitis $\mathrm{C}$ virus-infectes pacients receiving interferon alfa $20 \mathrm{~b}$ (IFN/RBV therapy). In: THE HEPATITIS single topic Conference, June, 2001, Chicago, Illinois.

WANG, G. L.; SEMENZA, G. L. General involvement of hypoxia inducible factor response to hypoxia. Proceedings of National Academy of Sciences of USA, Washington, v. 90, n. 9, p. 4304-4308, may 1993. doi: 10.1073/ pnas.90.9.4304

WANG, Y. J. et al. PEGylation markedly enhances the in vivo potency of recombinant human non-glycosylated erythropoietin: a comparison with glycosylated erythropoietin. Journal of Controlled Release, Amsterdam, v. 145 , n. 3 , p. $306-313$, aug. 2010 . doi: $10.1016 /$ j.jconrel.2010.04.021

WEISS, M. J. New Insights into erythropoietin and Epoetin Alfa: mechanisms of action, target tissues, and clinical applications. The Oncologist, Dayton, v. 8, suppl. 3, 
p. 18-29, dec. 2003. doi:10.1634/theoncologist.8-suppl_3-18

WINEARLS, G. C. et al. Effect of human erythropoietin derived from recombinant DNA on the anemia of patients maintained by chronic hemodialysis. The Lancet. London, v. 328 , n. 8517 , p. $1175-1178$, nov. 1986. doi: $10.1016 /$ S0140-6736(86)92192-6

WHO - WORLD HEALTH ORGANIZATION. Drug Information, v. 24, n. 1, 2010. Disponível em: <http:// www.who.int/medicines/publications/druginformation/ DrugInfo 2010_Vol24-1.pdf>. Acesso em: 13 mar. 2012.

YANG, W. S. et al. Darbepoetin alfa suppresses tumor necrosis factor- $\alpha$-induced endothelin-1 production through antioxidant action in human aortic endothelial cells: Role of sialic acid residues. Free Radical Biology \& Medicine, Oxford, v. 50, n. 10, p. 1242-1251, may 2011. doi: 10.1016/j.freeradbiomed.2011.02.005

ZANICHELLI, M. A. et al. Hematopoese, fatores de crescimento e aplicação clínica da Eritropoetina na Anemia da Prematuridade. Pediatria, São Paulo, v. 17, n. 2, p. 123-142, mai. 1995.

ZANJANI, E. D. et al. Studies on the liver to kidney switch of erythropoietin production. The Journal of Clinical Investigation, New Haven, v. 67, n. 4, p. 1183-1188, apr. 1981. doi: 10.1172/JCI110133 
Para publicar na revista Universitas:

Ciências da Saúde, acesse o endereço eletrônico www.publicacoesacademicas.uniceub.br.

Observe as normas de publicação, para facilitar e agilizar o trabalho de edição. 